\title{
MEASUREMENTS OF THE BETATRON FUNCTIONS IN RHIC
}

\author{
D. Trbojevic, L. Ahrens, M. Bai, V. Ptitsyn, T. Satogata, and J. van Zeijts * \\ BNL, Upton, NY 11973, USA
}

\begin{abstract}
The Relativistic Heavy Ion Collider (RHIC) provides collisions of the fully stripped gold ions for four experiments. This report shows results from measurements of the betatron functions within the Interaction Regions (IR) as well as in the arcs in both "blue" and "yellow" rings. A single quadrupole excitation or the beam position monitors' RMS. values at injection are used to obtain the betatron amplitude function.
\end{abstract}

\section{INTRODUCTION}

RHIC consists of two identical three fold symmetric rings which provided collisions during the run in the summer of 2000 of the fully stripped gold ions. During this commissioning run the maximum energy was 70 $\mathrm{GeV} /$ nucleon. At two interaction regions (IRs), one at 8 o'clock where is the large detector "PHENIX" located, and at 2 o'clock where the smaller experiment "BRAMS" resides collisions were established at points with $\beta^{*} \sim 3 \mathrm{~m}$. At the 6 and 10 o'clock the minimum of the beta function was $\beta^{*} \sim 8 m$. The RHIC lattice is made of six arcs with twelve standard $\sim 90^{\circ}$ FODO cells between the IRs. The IRs are made of almost the same FODO cells with missing dipoles, to allow for zero dispersion at collision points. The IR tunable FODO cells also allow matching of the betatron functions between the high focusing triplets and the arc FODO cells. The expected values of the lattice functions in RHIC are presented in Table 1.

Table 1: Maximum Twiss Functions in RHIC at the IR

\begin{tabular}{|l|c|c|c|c|}
\hline Region & $\beta_{x}$ & $\beta_{y}$ & $\beta_{\min }$ & $D_{x}$ atIP \\
\hline IR 8 and 2 & 424.94 & 413.76 & 8.50 & -0.0001 \\
IR 6 and 10 & 154.47 & 171.08 & 3.19 & 0.0048 \\
Arcs & 47 & 48 & 10.92 & 1.89 \\
\hline
\end{tabular}

The maximum values of the betatron functions are within the strong focusing triplet quadrupoles around the two low $\beta$ IRs while the other values are presented within the arc FODO cells. The beam positions around the ring were measured with a total of 334 beam position monitors (BPMs) per one ring. Almost half of the BPMs (total of 160) are dual plane monitors. Each BPM is capable of measuring and recording the turn by turn positions of the center of the beams. In this report a measurement of the $\beta_{x}, \beta_{y}$, and $D_{x}$ will be presented and compared with the design.

\footnotetext{
${ }^{*}$ Work performed under auspices of the U.S. Department of Energy
}

\section{AMPLITUDE FUNCTIONS $\beta_{X}, \beta_{Y}$ MEASUREMENTS}

The amplitude betatron function measurements during the RHIC commissioning were obtained first from the injection oscillations taking the rms value. A measurement in the horizontal plane is presented in Fig. 1 together with the predicted values, while the vertical plane measurements is presented in Fig. 2.

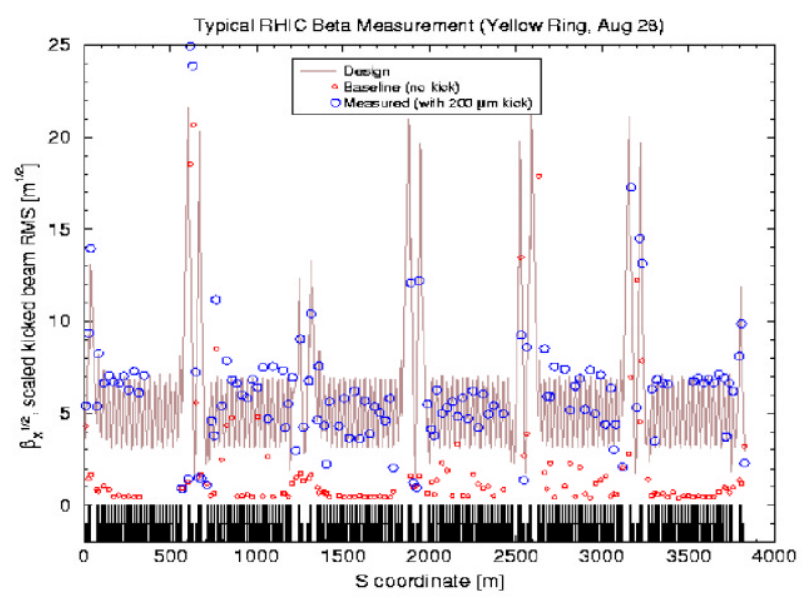

Figure 1: Measured -o- and predicted $\beta_{x}$ betatron function in the horizontal plane.

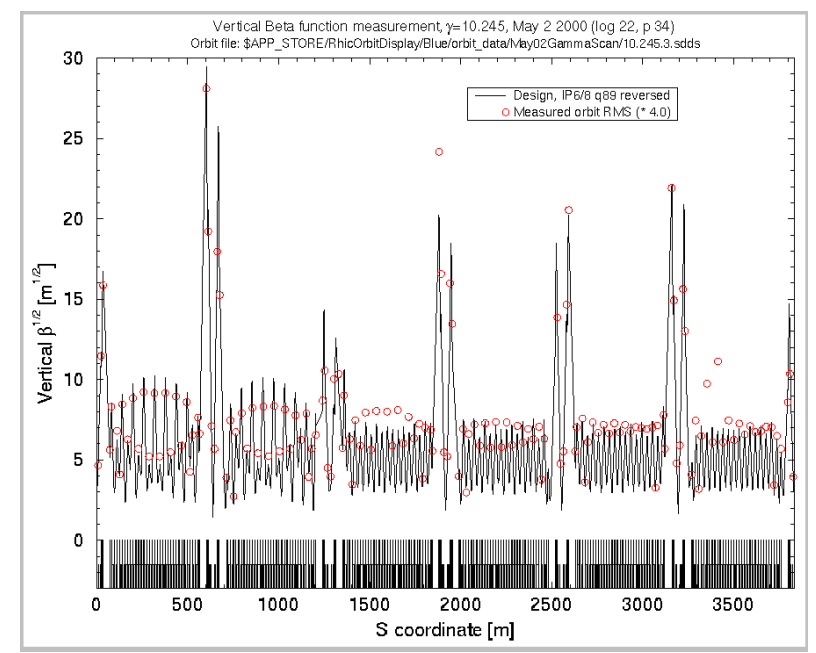

Figure 2: Measured -o- and predicted $\beta_{y}$ betatron function in the vertical plane. 


\section{DISPERSIONFUNCTION MEASUREMENTS}

At the beginning of the RHIC commissioning there was a clear disagreement between the measured and predicted horizontal dispersion measurements. With a help of the ON-LINE model a wrong polarity quadrupole power supply was detected. Three measurements of the dispersion function are presented in Fig. 3. The first measurement shown in Fig. 3 was done before a polarity of the quadrupole power supply was properly corrected. At the same plot design values are shown by the full line.

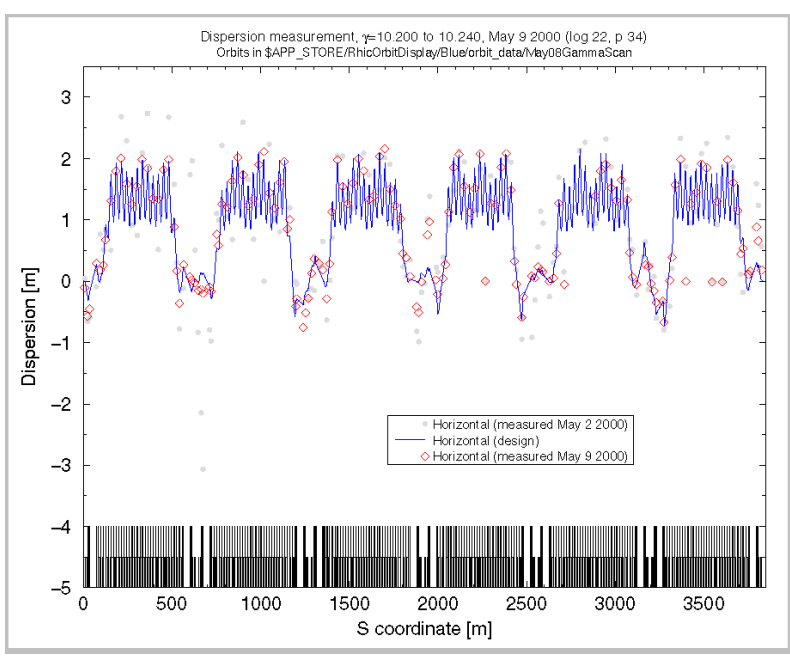

Figure 3: Dispersion function measurements before power supply correction - $\diamond$-, after the correction -o- and predicted horizontal and vertical dispersion functions.

\section{BETATRON FUNCTION MEASUREMENTSBY THETRIM QUADRUPOLES}

Although a special program was developed for a purpose of the different element polarity check it was used also for the betatron amplitude measurements. The betatron functions at each adjustable quadrupole are determined by measuring the tune shift [1] due to a change of strength $\mathrm{k} l$ as shown in Eq. 1

$$
\begin{gathered}
\Delta \nu \simeq \frac{1}{4 \pi} \int_{l} \beta(s) \Delta k d s, \\
\beta_{Q} \simeq \frac{4 \pi \Delta \nu}{l \Delta k},
\end{gathered}
$$

where $\beta_{Q}$ is the average function of the beta function at the quadrupole. The tune shift is measured by a betatron tune measurement system described above. A part of the application program control and results from the measurements are shown in Fig. 4.

The betatron functions at the trim quadrupole locations were measured at the trim quad locations around the ring as presented for the horizontal betatron function in Table 2

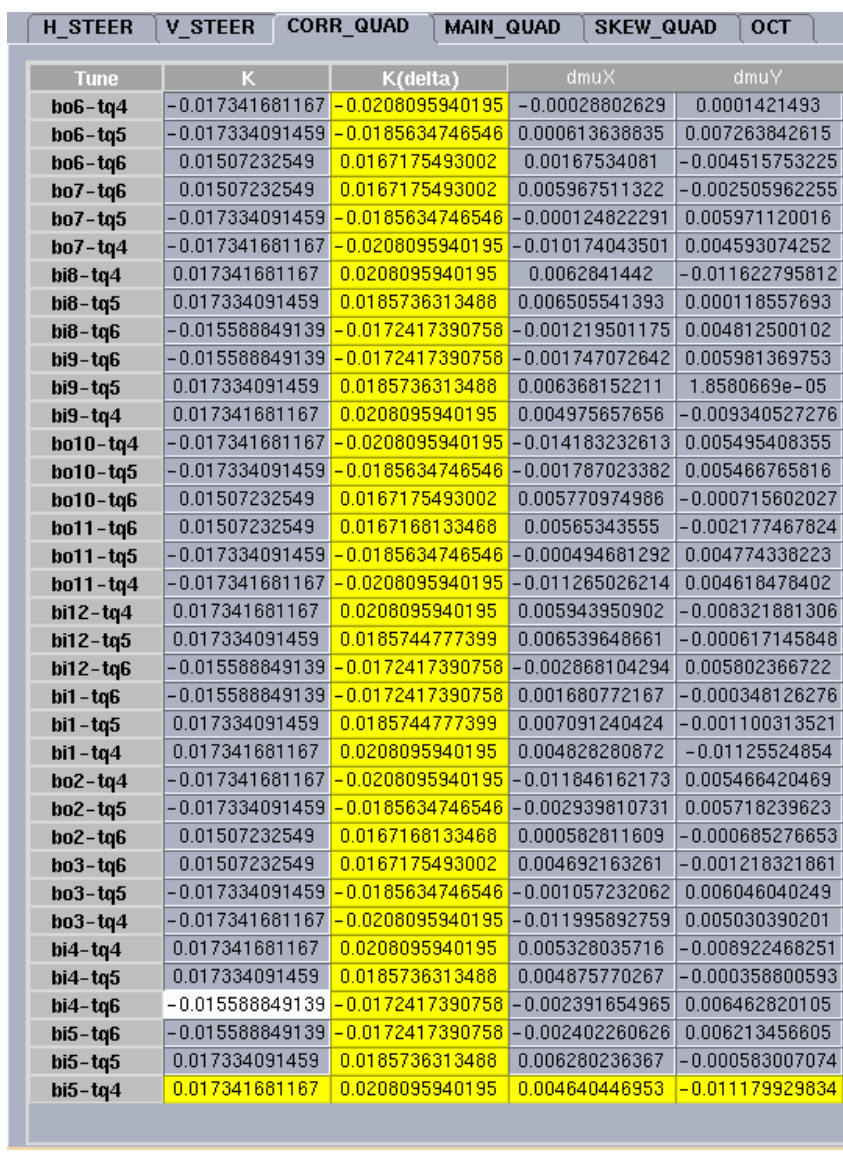

Figure 4: A part of a control page of the application program for the multi-element polarity check.

and for the vertical betatron function in Table 3. This is a part of the available data. The application had been built for finding elements with the wrong polarity during the commissioning and it is very easy to use. At the same time any discrepancies between the expected and measured values leads towards better understanding of the role of each element in RHIC.

Table 2: Horizontal Betatron function measurement

\begin{tabular}{|l|c|c|c|c|}
\hline Tr. quad & $\Delta \mathbf{~ k}$ & $\Delta \nu_{x}$ & $\beta_{x}$ & $\beta_{x}$ mod. \\
\hline bo2-tq4 & -0.00347 & -0.01185 & 42.93 & 39.28 \\
bo3-tq4 & 0.00347 & -0.01200 & 43.47 & 36.373 \\
bo3-tq5 & -0.00123 & -0.00106 & 10.80 & 7.68 \\
bo3-tq6 & -0.00165 & 0.00469 & 35.84 & 39.280 \\
bi9-tq4 & 0.00347 & 0.00498 & 18.03 & 18.9823 \\
\hline
\end{tabular}

\subsection{Difference Orbit Measurements}

One of the fastest methods of finding the correspondence between the measured beam position orbit positions and expected lattice functions is a single corrector dipole kick with recording the orbit differences. Results obtained by this method, shown in the other publications at this con- 
Table 3: Vertical Betatron function measurements

\begin{tabular}{|l|c|c|c|c|}
\hline Tr. quad & $\Delta \mathbf{~ k}$ & $\Delta \nu_{y}$ & $\beta_{y}$ & $\beta_{y}$ mod. \\
\hline bo2-tq4 & -0.00347 & 0.00547 & 19.8 & 18.87 \\
bo3-tq4 & 0.00347 & 0.00501 & 18.23 & 19.9 \\
bo3-tq5 & -0.00123 & 0.00605 & 61.77 & 64.50 \\
bo3-tq6 & -0.00165 & -0.00122 & 9.31 & 13.07 \\
bi9-tq4 & 0.00347 & -0.00934 & 33.85 & 36.46 \\
\hline
\end{tabular}

ference [2], were also used to find sources of the local decoupling.

\section{SUMMARY}

This report had shown betatron function measurement results during the commissioning run. These measurements had shown to be very useful during this period especially the dispersion function measurements which allowed fast error analysis using the on-line model. It is expected to commission in RHIC soon a new AC dipole system with possibility of a very fast betatron function measurements in the whole ring by looking the beam position monitor response in the turn by turn mode.

\section{REFERENCES}

[1] E.D. Courant and H.S. Snyder, "Theory of Alternating Gradient Synchrotron", Annals of Physics, Vol. 3, (1958), pp. $1-48$.

[2] V. Ptitsyn, J. Cardona, F. Pilat,J. P. Koutchouk, "Measurement and Correction of Linear Effects in the RHIC Interaction Regions", at this conference, RPAH122. 